\title{
Expression du mal-être dans des récits de vie de détenteurs de vidéos pédopornographiques
}

\author{
Frédéric Pugniere-Saavedra \\ Université Bretagne Sud, Laboratoire Prefics \\ frederic.pugniere-saavedra@univ-ubs.fr
}

\begin{abstract}
Résumé. À travers des récits de vie qui reconfigurent des réalités sociales vécues de locuteurs qui détiennent et qui diffusent des documents vidéos pédopornographiques, nous organiserons notre étude selon le continuum suivant : après une présentation de ce corpus dit sensible et du groupe de paroles en tant que dispositif mobilisé, nous aborderons le cœur de notre étude avec deux focales portant sur les régularités énonciatives d'une part et d'autre part sur les organisateurs de discours «que, mais» qui forgent la textualité de ce discours et qui participent à exprimer le mal être des locuteurs.
\end{abstract}

\begin{abstract}
Through narratives of life which re-configure social realities lived on speakers who hold and who spread documents child pornography videos, we shall organize our study according to the following continuum: after a presentation of this sensitive said corpus and the group of words as mobilized device, we shall approach the heart of our study with two focals concerning the enunciative regularities on one hand and on the other hand the organizers of speech "that, but " which make the textuality of this speech and who participate to express the evil to be speakers.
\end{abstract}

Cette contribution reposera sur l'analyse d'un corpus constitué de récits de vie qui dévoilent le locuteur, ils sont envisagés non pas en tant que «données factuelles » sur des trajectoires biographiques (Bertaux [1997] 2005 : 12-14) et des "points de vue subjectifs » sur ces données factuelles (Beaud $1996: 241$ ), mais en tant que reconstructions discursives du monde envisagées comme des mises en intrigue (Ricoeur 1983), comme des "opérations de configuration » qui procèdent à la transformation d'une multiplicité d'événements ponctuels et hétérogènes en " une histoire », cohérente et signifiante (ibid. p. 127). On envisagera ici le récit de vie ${ }^{i}$ en tant qu'activités (re)configurant les réalités sociales vécues des locuteurs par le choix des constructions singulières de la syntaxe, du lexique et de formes énonciatives (Nossik 2011).

Ces récits de vie ont été recueillis entre 2015 et 2017 dans le cadre de groupes de paroles (ce dispositif sera évoqué infra) de personnes pédophiles qui sont, rappelons-le, définies par l'OMS ${ }^{\text {ii }}$ comme étant « la préférence sexuelle pour les enfants, qu'il s'agisse de garçons, de filles, ou des deux, généralement d'âge pré-pubère ou au début de la pubertéiii $»$. Les locuteurs 
qui participent volontairement à ces groupes de paroles sont des victimes de pédophiles ${ }^{\text {iv }}$, des agresseurs en cours de jugement ou ayant fait l'objet d'un jugement, des détenteurs et consommateurs de pédopornographie, des hommes ayant des pulsions sans passage à l'acte, des proches d'agresseur et des pédophiles abstinents.

Nous nous intéresserons dans cette contribution uniquement aux personnes détentrices de vidéos à caractère pédopornographique ${ }^{\mathrm{v}}$ qui se racontent au travers du dispositif de groupe de paroles où les participants mettent en mots leur histoire familiale, transgénérationnelle, leur culture, leurs valeurs, leurs croyances et leurs propres trajectoires en lien avec la pédophilie ; le groupe de paroles constitue une "norme d'écoute " ${ }^{\mathrm{vi}}$ (Fassin 2004, Bass et Caevel 2005) au travers de laquelle les consommateurs de pédopornographie, par les configurations spécifiques de leur récit, expriment leur mal-être.

\section{1. Éléments de cadrage dans ce type particulier de discours axé sur le dévoilement de soi.}

\subsection{Configuration socio-interactionnelle particulière de ce corpus}

Parler de soi est une activité difficile pour de nombreuses raisons : risque de transgresser la "loi de modestie" ; risque surtout de "s'exposer" en se confiant, de se rendre vulnérable en "s'ouvrant" à autrui et en donnant accès à ses "réserves" (Goffman), ou même à ses secrets. Comme le souligne Vion (1994 : 217) « conventionnellement masquée, rarement évoquée et partagée, la souffrance ne se dit pas (loi de discrétion) et la décrire est pour le sujet une tâche de quasi-improvisation langagière ». Se dévoiler et se raconter relève d'un domaine à risques d'où l'abondance de formes de modulation (processus tendant à diminuer la part de subjectivité que chacun peut investir dans l'interaction: « registres de l'euphémisme, de l'atténuation, des circonlocutions, du discours précautionneux, des lexicalisations prudentes, des actes indirects, des préliminaires, des justifications, des auto-corrections, etc. » (Vion, 1992-2000:244) et de tension (processus inverse: «registres de l'hyperbole, de l'emportement, des lexicalisations marquées et pittoresques, des actes directs, etc. » (ibid)). Parler de soi au travers de groupes de paroles est également un contrat de communication (Charaudeau 1984) particulier. Les participants à ce groupe de paroles se réunissent une fois par mois au domicile de la présidente de l'association ${ }^{\text {vii }}$. Quinze personnes environ partageant une expérience en lien avec la pédophilie en arrière-plan sont réunies pendant trois ou quatre heures le samedi après-midi autour d'une grande table avec boissons, fruits et pâtisseries. Les participants sont plutôt décontractés, certains sont à l'aise pour parler d'eux, d'autres ont plus de difficultés pour mettre en mots leur histoire mais la convivialité (et parfois l'humour un peu douteux) semble faciliter leur récit. Il faut également noter qu'un participant peut venir plusieurs fois en fonction de son histoire, de son évolution (ou de sa non-évolution), de la temporalité de l'action judiciaire (cf. exemples 1).

\section{Exemples 1}

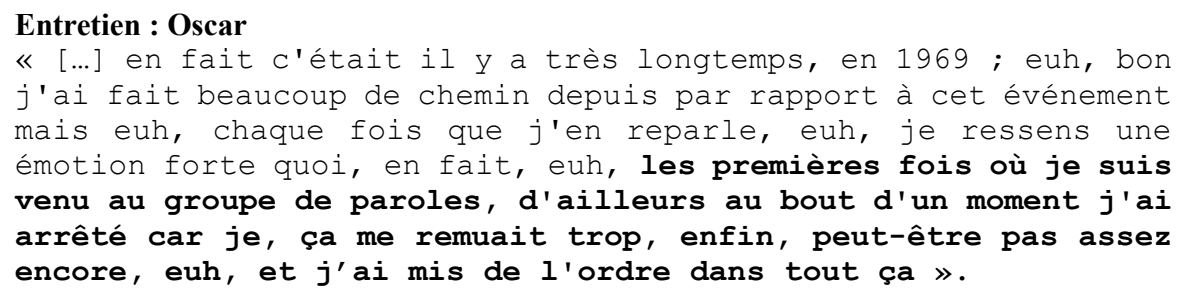

Á côté de cette grande table se trouve un espace accueillant environ huit observateurs (des étudiants, des psychologues, les membres de la famille des participants, des journalistes, des universitaires...) qui ne prendront en aucun cas la parole. 
Du point de vue interactionnel, la présidente de l'association endosse le rôle de modératrice, elle s'inscrit dans une fonction particulière et structurante et non dans un discours de maître, elle connait l'histoire de chaque participant ${ }^{\text {viii }}$ et leur donne la parole de façon à ce que chacun puisse rebondir sur l'histoire de l'autre. Le/la locuteur(rice) se raconte librement, il/elle ne reçoit au préalable aucune consigne de temps, ni de mise en récit et le/la locuteur(rice) met l'accent sur ce qui lui semble important.

La situation interactionnelle est complexe : le locuteur se raconte à des tiers présents, Charaudeau (1984: 112), (la présidente modératrice, les locuteurs qui se trouvent dans une situation comparable [partage d'expériences de victimes], les locuteurs qui ont un "profil » autre [agresseurs s'exprimant devant des victimes par exemple] et le groupe dans son ensemble) et à des tiers absents (absents parce que les observateurs ne peuvent prendre part aux échanges dialogaux, mais ils sont bien là physiquement et sont témoins de ce qui est dit). Ce groupe de paroles, où chacun doit écouter l'autre et prendre à son tour la parole, a pour objectif de trouver dans l'écoute du soutien parfois nécessaire, de faire se rencontrer des pédophiles, des victimes et des consommateurs de vidéos pédopornographiques pour que certains parviennent à se décentrer pour aller vers les autres ${ }^{\mathrm{ix}}$, ces locuteurs montrent aux autres participants qu'ils ont des ressources intérieures pour comprendre (et éventuellement affronter) l'Autre qui peut représenter par exemple la figure de l'agresseur pour une victime.

\section{Exemples 2}

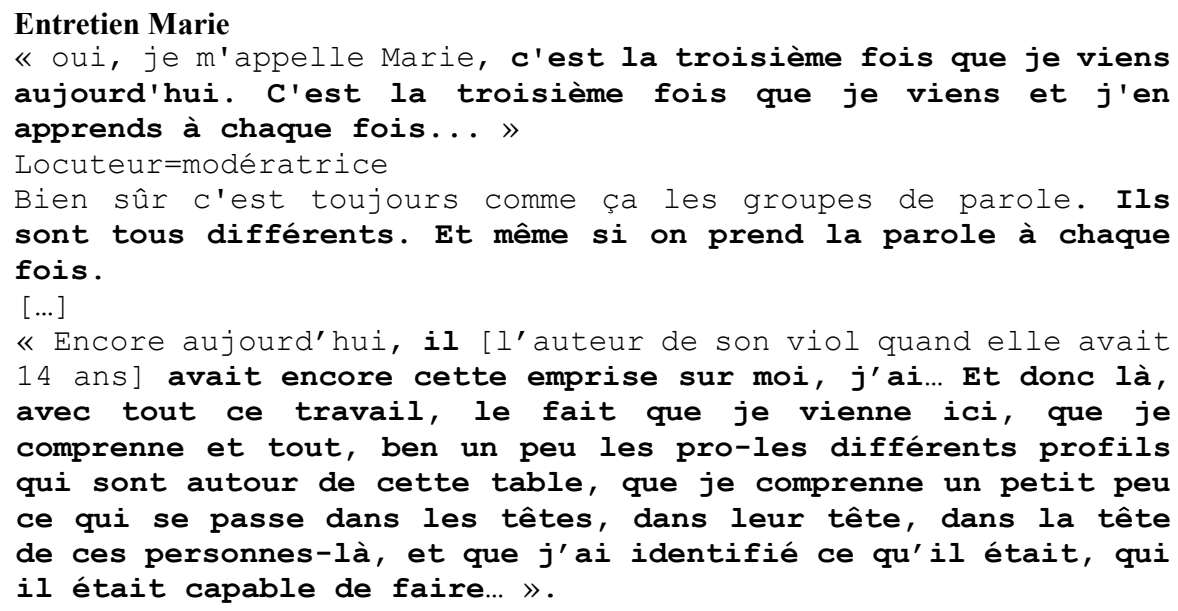

Ce dispositif comporte toutefois des limites. Il s'agit d'un genre de discours où les locuteurs sont totalement libres, ce discours est loin de la forme d'un entretien individuel (directif, semi-directif) que l'on pratique en sociologie ou en sociolinguistique. La modératrice sollicite peu la reformulation du locuteur, demande peu de rebondir sur les changements personnels consécutifs au viol ou à la diffusion de documents à caractère pédopornographique par exemple et demande peu de verbaliser de manière introspective les conséquences sociales des actes par exemple. Le lieu (le domicile de la présidente de l'association) facilite-t-il la prise de parole ? La libère-t-il ? Enfin et surtout, ne participent à ce groupe de paroles que les personnes dont la présidente a jugé que le groupe de paroles pourrait leur apporter quelque chose et que cette personne pourrait apporter quelque chose aux autres. Le rôle de filtre de la présidence n'exclut pas les agresseurs qui ont fait un travail sur eux-mêmes et qui, de ce fait, alimentent les échanges du groupe de paroles, mais exclut totalement les pédophiles pervers multirécidivistes. 


\subsection{Conditions technologiques favorables faisant émerger de nouveaux usages dans la diffusion des vidéos à caractère pédopornographique}

Le taux d'équipement « ordinateur-tablette-smartphone » est en hausse ${ }^{\mathrm{x}}$. L'équipement en ordinateur est relativement stable depuis $2012(82 \%)$ tandis que la proportion de connexion à internet à domicile affiche une progression continue $(85 \%,+2$ points $)$. Ce sont surtout les équipements nomades, tablettes tactiles et smartphones, qui se démocratisent à un rythme soutenu, respectivement +5 points et +7 points en 2016. Quatre Français sur dix sont désormais équipés en tablette et $65 \%$ ont un smartphone, ce qui implique de nouveaux usages d'internet en mobilité et l'essor des pratiques digitales depuis un téléphone mobile.

Parallèlement à ce taux d'équipement, le web 2.0 (web dit interactif ou web social) regroupe «sous la même enseigne de grandes variétés de dispositifs tels que les blogs (Bloggers, Thumbler) les Wikis (Wikipédia, Wikitravel), les sites de réseaux sociaux numériques (Facebook, Linked'in), les microblogues (Twitters, Jaiku), le bookmarking collectif, le partage de contenus médiatiques comme la photo, la musique et les vidéos » (Proulx et al 2012:4).

Enfin le fort taux d'équipement et les nouveaux usages sont dopés par la diffusion du très haut débit mobile ( $42 \%$ des possesseurs d'un téléphone mobile déclarent utiliser la 4G, une proportion multipliée par trois en deux ans (14\% en 2014) et la baisse des prix des fournisseurs d'accès à internet ont favorisé l'accès dans la sphère privée aux sites pornographiques «Indépendamment des difficultés à quantifier la présence de la pornographie sur internet, on peut du moins affirmer qu'elle est importante, il n'y a pas désaccord sur la question » (Dubois $2014: 48$ ) et des sites pédopornographiques ${ }^{\mathrm{xi}}$ avec en particulier l'émergence du grooming qui désigne les personnes qui, via les messageries instantanées, entrent en contact avec des mineurs en se faisant passer ou non pour un jeune du même âge, afin de le manipuler dans l'optique de lui faire commettre des actes à caractère sexuel.

\section{De la constitution du corpus au cadre théorique mobilisé}

\subsection{Corpus dit sensible et conventions de transcription}

Ce corpus sensible comporte environ 70 heures d'enregistrement. Le corpus dit sensible est à notre connaissance émergeant (Paveau et Pérea 2012/2015) et ne figure pas dans les habitus institutionnalisés de la recherche (Maingueneau 1991:11) ; il s'oriente principalement vers ce qui relève des problèmes de genre ou/et de sécurité (santé publique, sécurité intérieure, cyberdéfense, confidentialité des données sensibles, militaires, nucléaires...). Il se manifeste également par sa charge d'interdits, ce qui questionne nécessairement le chercheur par rapport à sa pratique et à son objet (cf. Les travaux F. Perea ${ }^{\mathrm{xii}}$, de $\mathrm{B}$. Damian ${ }^{\mathrm{xiii}}$ ) ; les travaux de Paveau portent sur le discours pornographique et pose comme principe que :

«Parler de la pornographie, c'est envisager les textes et les paroles sous l'angle de leur forme, et par conséquent décrire les mots, les expressions et les manières de dire, mais aussi les traditions textuelles, la construction des scripts et la manière dont ces discours sont situés, qu'ils soient ignorés, admis, ou rejetés dans l'ensemble des discours sociaux (2014:25).

Ce corpus d'enregistrement est intégralement transcrit et nous avons adopté les conventions de transcriptions qui ont été employées pour le projet CFPP2000 (Branca-Rosoff et alii [12$14])^{\text {xiv }}$ car nos préoccupations en termes d'analyse et de traitement du corpus nous semblaient proches de ce projet.

«Toute transcription est un compromis forcément boiteux entre le respect des particularités orales et la lisibilité. Puisque le corpus permet de revenir à la version orale, nous avons adopté une transcription orthographique afin de faciliter la lecture cursive et de simplifier l'exploitation des logiciels de concordance. Le code de 
transcription adopté est pour l'essentiel celui du DELIC ${ }^{\mathrm{xv}}$ qui a été légèrement adapté. Nous transcrivons les mots en orthographe sans correction des écarts à la norme quand ils correspondent à un morphème attesté en français ».

Le corpus se compose exclusivement d'hommes ${ }^{\mathrm{xvi}}$ lesquels se répartissent en trois groupes selon l'AFC (Analyse Factorielle des Correspondances) ci-dessous : les membres du groupe 1 (Édouard et Tom) mettent en avant la difficulté dans leurs relations aux femmes, dans leur besoin de consommer du cannabis et dans leur regard porté sur les enfants qui sont les seuls à être bienveillants à leur égard ; les membres du groupe 2 (Albert et Alain) mettent en avant le temps passé sur Internet et leur incompréhension à admettre le problème que constitue leur attirance pour les enfants, et les membres du groupe 3 (Thomas, Louis et Jules) remettent en question la désignation de pédophile du fait qu'ils sont intéressés par les images de jeunes filles sans qu'il n'y ait jamais eu agression (cf. tableaux de spécificités positives et négatives en annexes et les tableaux de fréquences pour les substantifs et les verbes).

\begin{tabular}{|c|c|}
\hline Locuteur anonyméxvii & nombre de formes \\
\hline Alain & 1025 \\
\hline Albert & 549 \\
\hline Jules & 663 \\
\hline Thomas & 510 \\
\hline Édouard & 1591 \\
\hline Tom & 262 \\
\hline Louis & 830 \\
\hline
\end{tabular}

Tableau 1 : répartition des locuteurs en nombre de formes

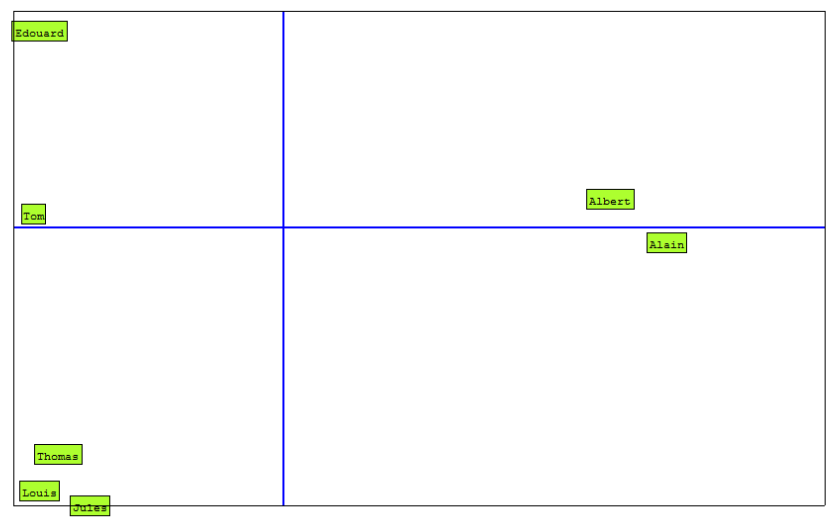

Figure $1:$ AFC des locuteurs

\subsection{Cadrage conceptuel}

On considérera que la parole repose sur le produit social d'une langue, mais en affiche l'usage individuel (Dubois et al., 1994 : 267), qu'elle relève du discours tel que le définit Foucault (1966), à la fois langage et matérialité, symptôme et signe, surface et profondeur et qu'en rassemblant mots, objets et pratiques (Mol, 2009 : 30), la parole ébauche une logique de soins parce qu'elle renvoie à la volonté de " s'engager dans des activités pratiques » (Mol, 2009 : 148). La parole constitue un socle partagé parmi les participants aux groupes de paroles, ce qui permet de l'appréhender comme un analyseur légitime des mécanismes de l'expérience du locuteur. Nous considérons dans le cadre de ce travail que la parole prononcée par les participants, puis transcrite et anonymée sera désignée par le terme de discours. Nous nous appuierons sur la conception du discours de Paveau pour qui : «Les discours ne sont pas 
seulement des suites de mots et de phrases, mais constituent des lieux où se définissent les normes et les valeurs, les prescriptions et les interdits, les goûts et les dégoûts, les qualités et les défauts, les identités, les légitimités, les gloires et les hontes » $(2014: 25)$. Les productions discursives des locuteurs seront appréhendées sous le prisme de l'analyse de discours (tendance française) qui prend appui sur les marques d'énonciation et qui considère qu'il existe des invariants, des propriétés spécifiques linguistiques et discursives pour rendre observables des configurations spécifiques de ce type de récits. La méthodologie d'analyse de discours sera complétée par une approche visant à faire ressortir la dimension argumentative de ces récits et par une approche textométrique qui propose une approche instrumentée des corpus, articulant synthèses quantitatives et analyses à même le texte (Lebart \& Salem 1994).

Mayaffre (2007 : 9) précise : «Encore moins que la fréquence d'un mot, la récurrence de segments ne peut être naïvement attribuée au hasard: soit elle pointe une contrainte syntaxique, soit elle indique une détermination ou option sémantique », il poursuit en 2012 en affirmant que la textométrie permet :

- «d'analyser un grand nombre de documents textuels que ni l'œil ni la mémoire ne sont capables de prendre en compte dans une telle marée de mots;

- de mettre en perspective les caractéristiques qui ne se révèlent que par rapport à un ensemble plus vaste;

- d'atteindre la précision, la systématicité, l'exhaustivité ou l'objectivité nécessaire pour une description scientifique;

- d'ouvrir des voies de l'interprétation historique loin de celles induites par l'actualité contemporaine, nos a priori, nos sujets favoris puisque l'outil ne ressent lui ni préférence, ni aversion » (2012: 14-16).

\section{Mise en mots du mal-être des consommateurs de vidéos pédopornographiques}

Les histoires personnelles des locuteurs s'inscrivent dans une quête, dans une recherche d'information pour comprendre ce qui les a fait basculer dans ce type d'attirance pour ces images. Deux types de régularités seront abordées ici : les régularités énonciatives (3.1) et deux marqueurs « que » et « mais » qui forgent la textualité de ce type de discours (3.2).

\subsection{Focale sur les régularités énonciatives saillantes}

Les histoires personnelles s'inscrivent dans une construction déictique où celui qui prend la parole s'exprime en tant que sujet parlant empirique (homme/femme qui formule des énoncés à travers sa voix...), en tant que locuteur qui produit un discours (personne invitée par la Présidente de l'association qui parle en son nom) et en tant qu'énonciateur (personne ayant, par sa trajectoire, le statut de possesseur et consommateur de vidéos pédopornographiques) qui est responsable de ce discours (Ducrot 1983). On parlera désormais d'énonciateur qui non seulement, par les marques de premières personnes [Je, me, moi (1360 occurrences)], assume ses propos mais construit une surenchère réaffirmée par le procédé d'emphatisation avec le pronom tonique renforçant le pronom personnel sujet [moi je (31 occurrences)].

\section{Exemples 3}

Entretien Édouard

Mais, tout ça pour dire que finalement, les enfants moi je les supporte pas, en vrai.

\section{Entretien Louis}


Et moi je me voyais comme un monstre, je me voyais comme le monstre qui était à la recherche d'enfants

À ce phénomène énonciatif s'ajoutent les phénomènes de nomination particuliers. On perçoit que nommer ne se limite pas à « représenter » le monde tel qu'il est, les faits tels qu'ils sont, mais à donner un point de vue, à prendre une position sur l'objet en choisissant de la désigner ou de la caractériser d'une certaine façon en y mettant de l'évaluation, de la distance, de la domination, de la discrimination, de la proximité, du jugement selon la perception que l'on en a (Siblot 1998). Les désignations du consommateur de vidéo (cf. tableau $n^{\circ} 2$ ) se répartissent entre hyperonyme (agresseur) et hyponymes (pédophile, délinquant sexuel, abuseur) objectifs et désignations subjectives (monstre, cybercriminel, criminel). Certaines désignations du mineur sont objectives (jeune-fille, garçon, mineur, victime), d'autres plus subjectives (gamin(e)) et les désignations de l'acte déviant et ses co-référents renvoient volontairement à un espace temporel vague (Brunner 2014) sans bornes de début ou de fin mais construit avec un déictique (mon histoire, cet évènement, dans mon souvenir); à des désignations abstraites omnisignifiantes (truc) et à des pronoms cataphoriques (ça).

\begin{tabular}{|c|c|c|}
\hline $\begin{array}{l}\text { Auto-désignations du } \\
\text { consommateur de vidéo } \\
\text { pédopornographique }\end{array}$ & Désignation du mineur & $\begin{array}{l}\text { désignations de l'acte avec } \\
\text { ses Co référents }\end{array}$ \\
\hline $\begin{array}{r}\text { Pédophile(s) (38), monstre (6), } \\
\text { agresseur (4), délinquant sexuel } \\
\text { (4), abuseur (2), cybercriminel (2), } \\
\text { criminel (2). }\end{array}$ & $\begin{array}{l}\text { Enfant(s) } 71, \text { fille (s) } 63, \\
\text { petite fille (31) jeune(s) 28, } \\
\text { gamin(s) 16, gamine(s) 12, } \\
\text { victime 11, garçon(s) } 9, \\
\text { mineur 3, fillette(s) 2. }\end{array}$ & $\begin{array}{l}\text { ça }(513) \text {, truc (35), histoire } \\
\text { (4), cet événement ( } 7) \text {, acte } \\
\text { (7), souvenir (2). }\end{array}$ \\
\hline
\end{tabular}

Tableau 2 : Désignations signifiantes

L'énonciateur met ainsi en mots la manière dont il renvoie aux circonstances qui l'ont fait basculer dans la pédopornographie en mobilisant des désignations vagues, ce qui évite de les nommer explicitement (cf. exemples 4).

\section{Exemples 4}

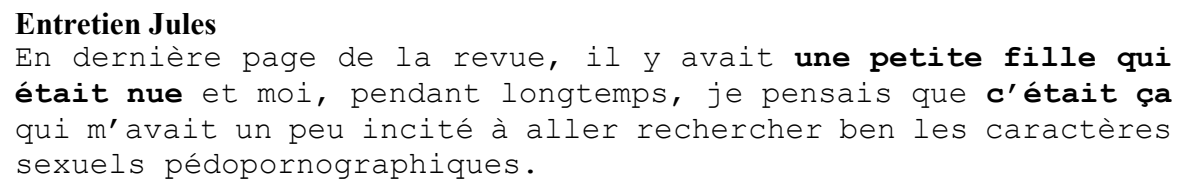

\section{Entretien Alain}

Je veux dire, en fait notre relation n'a pas changé de ce qu'elle était avant, avant cet événement. Donc à partir de ce moment-là j'ai vraiment pu faire un vrai travail qui maintenant m'a...aidé.

Les désignations à la fois signifiantes (autodésignation et désignation de l'enfant) et vagues (désignation d'un moment considéré comme point de référence ou de conscientisation) construites avec les marques énonciatives de personnes posent le discours comme complètement assumé par le locuteur, ce qui participe à réaffirmer, à rendre acceptable et utile pour lui et pour les autres sa participation au groupe de paroles.

\subsection{Focale sur deux organisateurs de discours}

Nous aborderons dans cette section les deux organisateurs de discours les plus fréquents (que et mais) ${ }^{\text {xviii }}$ qui forgent la textualité (ce qui fait d'un texte une suite linguistique signifiante « un ensemble cohérent progressant vers une fin et parvenant à constituer une complétude de 
sens » [Détrie, Siblot, Verine $2001: 349])$ et qui participent à expliquer la relation causale qui les lie à leurs actes. La dimension argumentative du discours mobilisée ici ne vise en rien à nous convaincre que les locuteurs sont ou non des agresseurs ou à nous persuader qu'ils disent la vérité. Nous nous situerons dans la perspective élargie ${ }^{\mathrm{xix}}$ de la définition de l'argumentation défendue par R. Amossy (2000:37) constituée par «les moyens verbaux qu'une instance de locution met en œuvre pour agir sur son allocutaire en tentant de la faire adhérer à une thèse, de modifier ou de renforcer les représentations et les opinions qu'elle leur prête, ou simplement d'orienter leurs façons de voir ou de susciter un questionnement sur un problème donné ».

\subsubsection{L'organisateur de discours que}

Une exploration du corpus par les connecteurs montre que les récits personnels font l'objet syntaxiquement d'une forte nominalisation ou d'adjectivation à travers le que (790 occurrences) (cf. fig. ${ }^{\circ} 2$ ) qui renvoie sémantiquement à l'expression de la justification du locuteur dans son dire (que je, parce que).

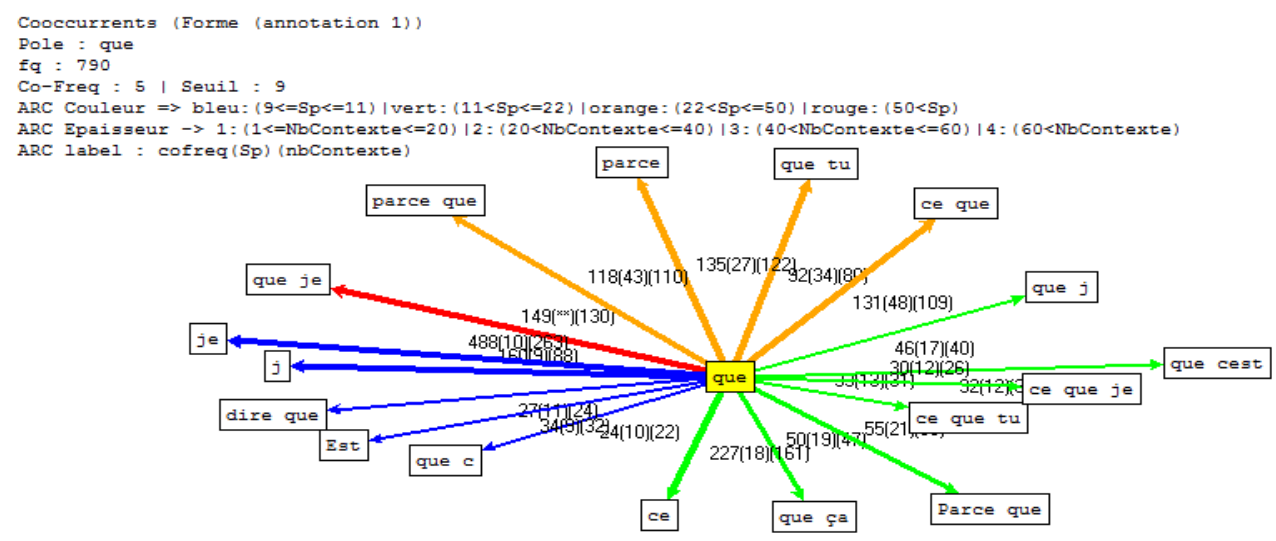

Figure 2 :Répartition de Que

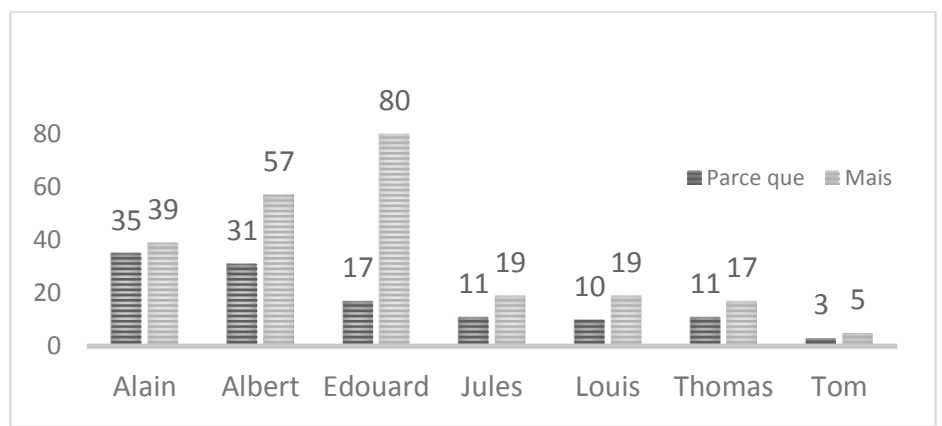

Figure 3 : Répartition des connecteurs les plus fréquents par locuteur 
Trois principaux types d'arguments sont mobilisés pour apporter une explication justifiant à la fois leur détention de vidéos pédopornographiques et leur inclination pour la pédophilie.

L'argumentation par l'investigation causale. En général, plusieurs causes sont suggérées ou évoquées; dans leur récit, les locuteurs manifestent une dépendance (au cannabis, au naturisme, au porno, à la technologie informatique principalement) pour aborder le moment où la justice sévit (saisie des ordinateurs, plainte, détention provisoire et incarcération).

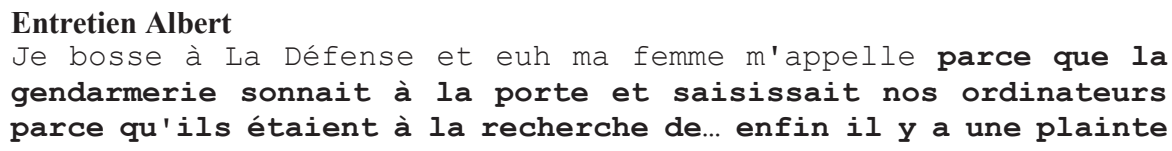

\section{Entretien Thomas}

Je consommais vachement de cannabis, en fait... avec le temps je me suis rendu compte que c'était simplement parce que je voulais fuir mes problèmes [...] Maintenant je joue encore beaucoup en ligne mais j'ai plus le même plaisir parce que c'est juste pour fuir en fait.

\section{Entretien Jules}

Comme d'autres ici, je suis entré dans le milieu de la pédopornographie en fréquentant d'abord les publications papier puis les sites internet de naturistes parce que $j^{\prime}$ aimais voir les enfants à poil, ça m’excitait.

L'argument par la réfutation du lien causal. Cette forme de réfutation permet au locuteur de faire porter à l'institution pénitentiaire actuelle une partie des conséquences.

\section{Entretien Alain}

Éviter la prison c'est important parce que je ne pense pas le milieu carcéral, euh, ça t'aide pour t'en sortir [...] Enfin je me suis dit que j'étais vraiment dans la merde parce que je savais que j'étais en récidive, que je regardais trop de gamins à poils

L’argument par dévalorisation de soi.

\section{Entretien Thomas}

Si une fille elle vient, elle m'accoste, ben je vais limite la repousser, parce que pour moi ben je la mérite pas

\subsubsection{L'organisateur de discours mais}

Le récit des locuteurs s'inscrit également dans la construction d'un discours asymétrique (Ducrot 1980) exprimé par la conjonction mais (cf. infra fig. n³) qui renvoie à différentes valeurs sémantiques ${ }^{\mathrm{xx}}$ (opposition [dans $29 \%$ des cas], restriction [dans $22 \%$ des cas], réfutation [dans $7 \%$ des cas], justification [dans $5 \%$ des cas]) et explication [dans $4 \%$ des cas]; à différents aspects discursifs (marqueurs d'oralité [dans 15\% des cas, et de thématisation [dans $9 \%$ des cas]) à partir d'un terme (ou d'une locution) pivot pris comme point de référence. Dans la construction $x$ mais $y, y$ constituera un argument plus fort qui oriente vers une conclusion pressentie de l'auteur, au détriment de $x$. 


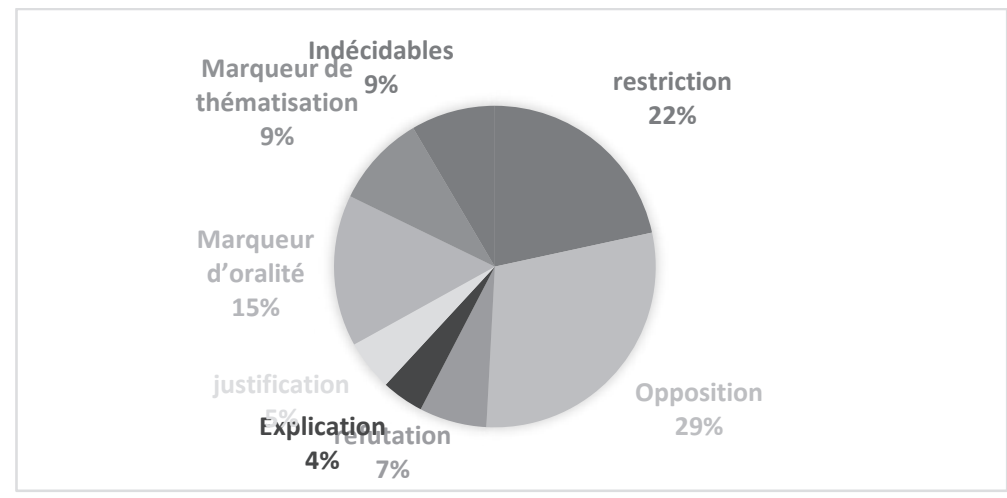

Figure 5 : Répartition sémantique de Mais

\section{Valeur d'opposition}

La conjonction mais construite avec un antonyme post posé (mineur $v s$ majeur) renforce ici le caractère déviant et participe à une prise de conscience du locuteur.

\section{Entretien Albert}

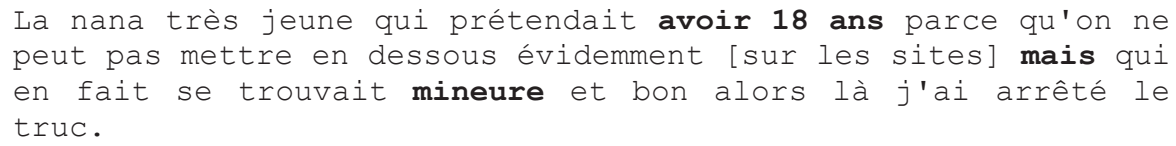

Dans l'exemple suivant avec l'opposition, (le patron vs les autres) la caractéristique principale des autres qui consiste en l'occurrence à ignorer l'activité d'Edouard renvoie à une sorte d'apaisement.

\section{Entretien Édouard}

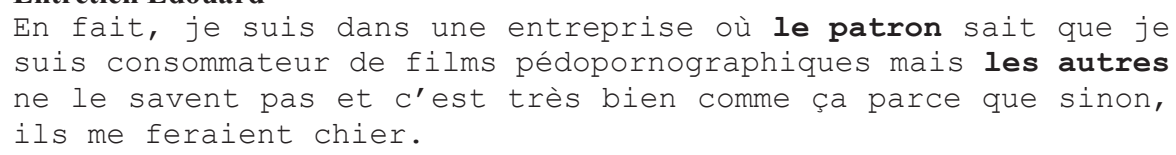

\section{Valeur restrictive}

La catégorie fille puis jeune fille constituera un positionnement moins fort de la part du locuteur que les plus petites (fille) de 10 à 13 qui est plus explicite dans cette situation de discours et qui est renforcée par le marqueur d'intensité mais surtout

\section{Entretien Alain}

J'ai toujours eu une attirance pour les filles, les jeunes filles, mais surtout pour les plus petites de 10 à 13 ans

\section{Valeur explicative}

Dans les deux exemples suivants, les valeurs des mots pivot moche ou suicide sont amoindries par des compléments positionnés après la conjonction mais construisant ainsi une explication particularisante.

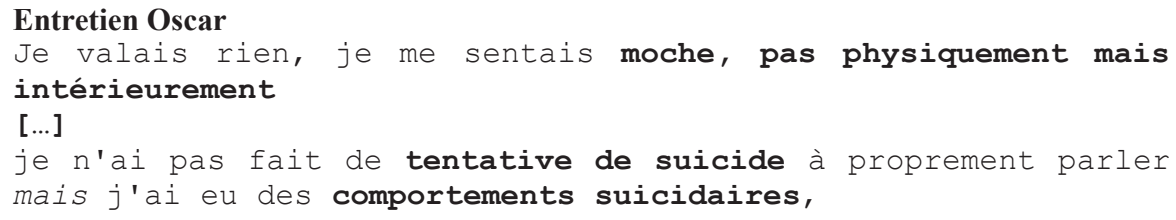


Dans les exemples suivants, la réfutation portant sur les tremblements alimente un positionnement argumentatif qui consiste à mettre l'accent sur l'intensité avec laquelle sont corps réagit.

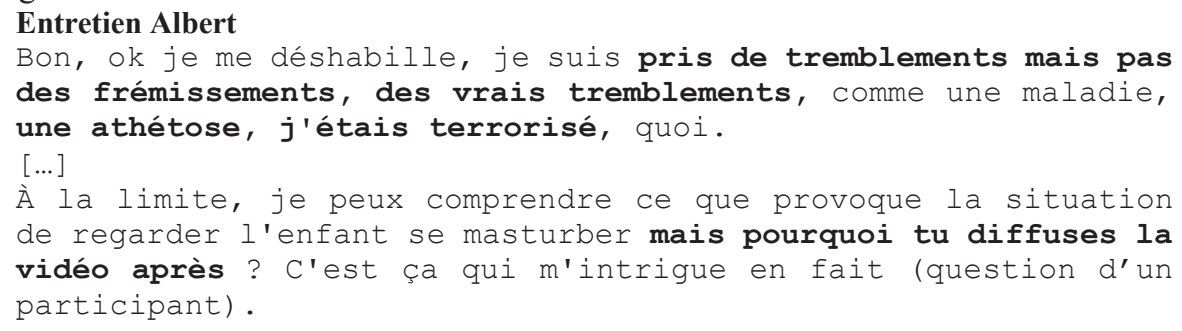

\section{Valeur justificative}

Le locuteur se justifie en minimisant la portée de ses actes de deux manières en considérant qu'il ne s'agit (que) de photos d'enfants nus, (le locuteur n'a alors pas l'impression de transgresser) et que dans un contexte naturiste, il y a une forme de normalité ou d'acceptabilité sociale.

Entretien Alain

C'était en fait que des photographies d'enfants à poil mais dans un milieu naturiste, c'était l'excuse on va dire, c'est vrai que ça m'excitait.

\section{Marqueurs de fermeture d'unité syntaxique à l'oral}

La conjonction mais construite avec l'adjectif bon et l'interjection heu (mais bon, mais euh) renvoient à un énoncé court qui alimente la fin d'une séquence.

\section{Entretien Alain}

J'aurais pris encore plus cher parce que justement je me serai caché derrière ce truc... exactement... Mais bon, ce qui devait arriver, arriva

\section{Entretien Édouard}

J'ai téléchargé des photos sur Internet, elle [sa femme] a raison je l'ai trompée. J'ai eu du désir pour d'autres personnes. Je me suis masturbé en pensant à d'autres personnes mais euh, je l'aime encore et c'est ça le principal, non ?

\section{Marqueur de thématisation}

La conjonction mais peut être un marqueur thématique qui reprend des référents qui ne sont pas strictement liées au cotexte mais qui dépend d'un accord référentiel partagé.

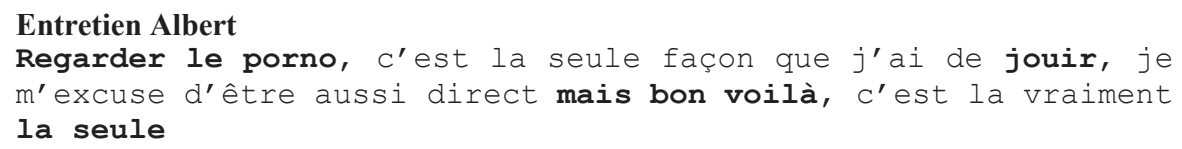

\section{Entretien Louis}

On parlait des personnes, des Anonymous sur Internet qui tendaient des pièges, mais voilà, ils faisaient payer les pédophiles, ils veulent se payer les pédophiles.

Ces 2 marqueurs construisent, à des niveaux différents du récit, une hétérogénéité de valeurs (surenchère, réfutation, opposition, restriction, justification, conséquence, emphase...) qui installent le locuteur dans une instabilité morale, laquelle se construit avec des marqueurs 
d'émotion lexicalement marqués vers l'expression d'un mal-être (Pugniere-Saavedra à paraître) avec de nombreux compléments de reprise.

\section{En guise de conclusion}

Parler de soi en tant que consommateur (voire de diffuseur) de vidéo pédopornographique, consiste avant tout à évoquer les circonstances (son histoire avec les enfants, sa relation à la sexualité entre adultes, sa relation aux jeux, à la technologie...) et la psychologie de l'énonciateur (la construction de sa sexualité, l'image de soi, l'assurance de soi...).

Pour appréhender ce discours, tandis que l'analyse de discours fait émerger les régularités (lexicales et sémantiques [(désignations, caractérisations], syntaxiques, énonciatives [marqueurs déictiques]) à travers ce dispositif particulier du groupe de paroles, la dimension argumentative du discours suscite, elle, un questionnement à la fois auprès des détenteurs de vidéos pédopornographiques que des participants au groupe de parole.

Les constructions énonciatives qui abordent les désignations des pédophiles et les constructions argumentatives induites par parce que et mais participent à agir sur l'allocutaire en tentant de l'aider à comprendre et à évaluer des mécanismes plus ou moins implicites. Instrumentaliser le naturisme, réfuter les conditions d'incarcération, désorienter l'interlocuteur en se présentant sous ses aspects vulnérables, minorer ses actes en mettant l'accent sur l'âge des enfants... participent-ils à rendre plus acceptables ces récits de vie ? Permettent-ils aux locuteurs une prise de conscience pour eux-mêmes et pour les autres?

\section{Références bibliographiques}

Amossy, R. (2000). L'argumentation dans le discours, Nathan Université, Nathan Université, Paris.

Barbet, D. et Mayaffre, D. (2007). «Débats pour l'Élysée », Mots. Les langages du politique [En ligne], 89 | 2007. URL : http://mots.revues.org/18733.

Bass, D. et Caevel (de), H. (2005). Au fil de la parole, des groupes pour dire, Eres, Paris.

Beaud, S. (1996). "L'usage de l'entretien en sciences sociales. Plaidoyer pour l'entretien ethnographique » Volume 9, 226-257.

Bertaux, D. (1997). Le récit de vie, Armand Colin, Paris.

Branca-Rosoff, S., Fleury, S., Lefeuvre, F. et Pires, M,. (2012), Discours sur la ville. Corpus de Français Parlé Parisien des années 2000 (CFPP2000).

Brunner, P. (2014). Le vague / die Vagheit : du mot au concept, pragmatique et folk linguistique, Lambert-Lucas, Limoges.

Chareaudeau, P. (1984). Aspects du discours radiophonique, Didier-Erudition, Paris.

Ducrot, O. (1980). Les mots pour le dire, les éditions de minuit, Col 1. Le sens commun.

Fassin, D. (2004). Des maux indicibles. Sociologie des lieux d'écoute, La Découverte, Paris, 198 p.

Foucault, M. (1966). Les mots et les choses: une archéologie des sciences humaines, Gallimard, coll. «Bibliothèque des sciences humaines », Paris, $405 \mathrm{p}$.

Kerbrat-Orecchioni, C, et Traverso, V. (2007). Confidence, dévoilement de soi dans l'interaction, Niemeyer, Tibingen.

Lebart, L. \& Salem, A. (1994). Statistique textuelle, Broché, Paris.

Maingueneau, D. (1991). L'Analyse du discours, Introduction aux lectures de l'archive, Paris, Hachette, Nouvelle édition mise à jour : l'Analyse du discours, Hachette.

Mayaffre, D. (2012). Nicolas Sarkozy. Mesure et démesure du discours Presses de Sciences Po, Références.

Mol, A. (2009). Ce que soigner veut dire : repenser le libre choix du patient, Paris, Presses des Mines.

Nossik, S. (2011). «Les récits de vie comme corpus sociolinguistique : une approche discursive et interactionnelle », Corpus, $10 \mid 2011,119-135$.

Paveau, M.-A. (2014). Le discours pornographique, La Musardine, Paris.

Paveau, M.-A. et F. Perea F. (coord.), (21012). « Sujets sensibles », Cahiers de praxématique, n59. 
Plantin, C. (2016). Dictionnaire de l'argumentation. Une introduction aux études argumentatives, ENS Edition, Paris.

Proulx, S. et Breton P. (2012). L'explosion de la communication Broché, La découverte, Paris.

Ricoeur, P. (1983). Temps et récit. Tome I : L'intrigue et le récit historique, Le Seuil, Paris.

Siblot, P. (1998). « Nommer, c'est déjà prédiquer », Cahiers de praxématique, 30, 37-54.

Vion, R. (1992). La communication Verbale. Analyse des interactions Hachette Supérieur, Paris.

Vion, R. (1994). «La linguistique et le dialogue », communication au colloque Dialogique, Université du Maine, (Peter Lang, Berne).

\section{Annexes}

\begin{tabular}{|r|l|r|c|}
\hline \multicolumn{5}{|c|}{ Entretiens Albert et Alain } \\
\hline \multicolumn{1}{|c|}{ item } & ind-spécif + & Item & $\begin{array}{c}\text { ind- } \\
\text { spécif- }\end{array}$ \\
\hline euh & 45 & lui & -5 \\
\hline ne (sais pas) & 30 & Enfin & -5 \\
\hline parce que & 29 & hein & -6 \\
\hline avais & 17 & Je & -7 \\
\hline étais & 14 & dit & -7 \\
\hline était & 14 & j'avais & -7 \\
\hline été & 8 & j'étais & -9 \\
\hline internet & 8 & Ben & -11 \\
\hline enfin & 7 & Mais & -11 \\
\hline avait & 6 & Et & -28 \\
\hline
\end{tabular}

\begin{tabular}{|r|l|r|l|}
\hline \multicolumn{5}{|c|}{ Entretiens Jules, Thomas et Louis } \\
\hline item & ind-spécif + & Item & $\begin{array}{c}\text { ind- } \\
\text { spécif- }\end{array}$ \\
\hline c'était & 16 & pas & -4 \\
\hline j'ai & 14 & envie & -4 \\
\hline euh... & 13 & Elle & -5 \\
\hline c'est & 11 & de & -5 \\
\hline ben & 9 & femme & -7 \\
\hline pédophile & 7 & euh & -14 \\
\hline mail & 7 & ne & -14 \\
\hline m'a & 7 & ai & -19 \\
\hline ouais & 6 & est & -23 \\
\hline
\end{tabular}

\begin{tabular}{|r|l|r|c|}
\hline \multicolumn{5}{|c|}{ Entretiens Tom et Edouard } \\
\hline \multicolumn{1}{|c|}{ item } & ind-spécif + & Item & $\begin{array}{c}\text { ind- } \\
\text { spécif- }\end{array}$ \\
\hline C'est & 24 & dire & -6 \\
\hline femme & 13 & était & -7 \\
\hline lui & 11 & étais & -8 \\
\hline Elle & 10 & je & -9 \\
\hline qu'elle & 9 & ne & -10 \\
\hline Ben & 9 & avais & -12 \\
\hline mais & 9 & est & -17 \\
\hline enfants & 7 & euh & -22 \\
\hline et & 7 & ai & -29 \\
\hline
\end{tabular}

\begin{tabular}{|r|l|r|l|}
\hline \multicolumn{5}{|c|}{ Entretiens Albert et Alain } \\
\hline fréquence & \multicolumn{1}{|c|}{ Subst. } & fréquence & \multicolumn{2}{|c|}{ verbe } \\
\hline 47 & ans & 77 & dire \\
\hline 22 & moment & 45 & faire \\
\hline 21 & truc & 42 & être \\
\hline $\mathbf{1 7}$ & enfants & 16 & avoir \\
\hline $\mathbf{1 5}$ & problème & 13 & voir \\
\hline 15 & femme & 7 & comprendre \\
\hline 15 & vie & 6 & partir \\
\hline 13 & personne & 6 & revenir \\
\hline 12 & mère & 5 & falloir \\
\hline 12 & parents & 4 & rentrer \\
\hline
\end{tabular}

\begin{tabular}{|c|c|c|c|}
\hline \multicolumn{4}{|c|}{ Entretiens Jules, Thomas et Louis } \\
\hline fréquence & Subst. & fréquence & verbe \\
\hline 28 & ans & 40 & dire \\
\hline 27 & fois & 28 & être \\
\hline 17 & filles & 19 & faire \\
\hline 15 & peur & 9 & voir \\
\hline 14 & moment & 8 & arrêter \\
\hline 14 & fille & 6 & regarder \\
\hline 13 & amis & 5 & consommer \\
\hline 11 & vidéos & 5 & travailler \\
\hline 10 & Internet & 4 & aider \\
\hline 10 & besoin & 4 & donner \\
\hline
\end{tabular}

\begin{tabular}{|r|l|r|l|}
\hline \multicolumn{5}{|c|}{ Entretiens Tom et Edouard } \\
\hline \multirow{2}{*}{ fréquence } & Subst. & fréquence & \multicolumn{2}{|c|}{ verbe } \\
\hline 43 & femme & 30 & être \\
\hline 31 & enfants & 26 & dire \\
\hline 25 & fois & 24 & faire \\
\hline 20 & ans & 21 & voir \\
\hline 18 & temps & 19 & avoir \\
\hline 18 & chose & 13 & aller \\
\hline 17 & choses & 12 & regarder \\
\hline 17 & moment & 6 & arrêter \\
\hline 14 & besoin & 5 & changer \\
\hline 11 & cannabis & 5 & revoir \\
\hline & & &
\end{tabular}

\footnotetext{
' On privilégiera ici le terme Récit de vie à celui de confidence qui étymologiquement renvoie à la fidélité, à la confiance que l'on accorde à celui à qui on se confie dans la vie privée ou intime. La confidence est, selon nous, un dévoilement de soi attendu dans certaines interactions, et même obligatoire dans certains contextes institutionnels (confessionnal, cabinet du médecin ou du psychanalyste, entretien d'embauche, témoignage au tribunal, etc.). Outre les confidences révélées dans l'espace privé, il y a celles divulguées dans l'espace public où le sceau du secret est rompu par le nombre de destinataires potentiel : les autobiographies, les mémoires, les interviews de politiques données dans des journaux, les confessions sur le divan d'un plateau télé, les confessions dans le confessionnal de certains programmes de télé réalité et plus récemment encore dans des blogs ou des sites de rencontres...La situation
} 


\begin{abstract}
interactionnelle sur laquelle nous travaillons est différente même si parfois ces récits de vie comportent à certains moments un dévoilement de soi.

ii Selon la Classification Internationale des Maladies (CIM) publiée par l'Organisation mondiale de la santé (OMS), la pédophilie est classée dans les Troubles de la préférence sexuelle (F65) qui eux-mêmes font partie des Troubles de la personnalité et du comportement chez l'adulte (F60-F69). La pédophilie (F65.4) est définie comme étant « la préférence sexuelle pour les enfants, qu'il s'agisse de garçons, de filles, ou des deux, généralement d'âge pré-pubère ou au début de la puberté ».
\end{abstract}

iii Notons que l'âge de la victime entraine une désignation différente :

Nepiophilie : préférence sexuelle pour les nourrissons et les tout-petits (généralement 0-12 mois).

Infantophilie : préférence sexuelle pour les tout-petits (1-6 ans).

Pédophilie : intérêt sexuel pour les enfants pré-pubères.

Hébéphilie un intérêt sexuel primaire pour les personnes pubères de 12-14 ans.

Ephebophilie : intérêt sexuel pour les adolescents.

iv Le corpus total est constitué de victimes [35\%], d'agresseurs [20\%] et de détenteurs et consommateurs de pédopornographie [26\%], d'homme ayant des pulsions sans passage à l'acte [4\%], de proches d'agresseur [3\%] et de pédophile abstinent [12\%]. Les trois premiers «profils » représentent à eux seuls $81 \%$ des participants à ces groupes de paroles.

${ }^{v}$ La pédopornographie est définie, selon la convention des droits de l'enfant, comme « Toute représentation, par quelque moyen que ce soit, d'un enfant s'adonnant à des activités sexuelles explicites, réelles ou simulées, ou toute représentation des organes sexuels d'un enfant, à des fins principalement sexuelles » et la cyber pédopornographie consiste en l'enregistrement, la possession et la diffusion d'images pornographiques représentant des mineurs via l'internet ». La pédopornographie est sanctionnée par l'article 227-23 du Code pénal. Le fait, en vue de sa diffusion, de fixer, d'enregistrer ou de transmettre l'image ou la représentation d'un mineur lorsque cette image ou cette représentation présente un caractère pornographique est puni de cinq ans d'emprisonnement et de 75000 euros d'amende. Lorsque l'image ou la représentation concerne un mineur de quinze ans, ces faits sont punis même s'ils n'ont pas été commis en vue de la diffusion de cette image ou représentation.

vi Les politiques sociales des années 1990 ont largement favorisé l'émergence des lieux d'écoute pour modérer les formes de souffrance par la libération de la parole dans un but thérapeutique (Fassin 2004). L'écoute fait l'objet d'un large consensus social et offre une réponse à la fois humaniste et immédiatement visible de l'action politique. Écouter l'Autre devient un acte. Et « cet intérêt manifesté pour les histoires des gens, au-delà des seules nécessités de la mission du dispositif, cette attention à leurs infortunes et à leurs misères s'inscrivent dans une manière d'aborder les relations sociales à travers une forme de sollicitude intimiste qui crée un espace de relations privilégiées. Il arrive parfois que ces lieux soient les seuls où des personnes puissent parler d'elles-mêmes et de ce qui les touche le plus. Ainsi se reconstituent, en dehors des cercles traditionnels de socialisation familiale, amicale, professionnelle ou de voisinage, des liens étroits totalement construits autour d'un rapport institutionnel - et néanmoins personnel - d'assistance et de solidarité » (2004:73).

vii http://www.ange-bleu.com/objectifs.php

${ }_{\text {viii }}$ La présidente assure une permanence par mails et par téléphone, elle reçoit tous les appels des locuteurs en détresse (victimes, agresseurs ou détenteurs et/ou consommateurs de pédopornographie...) et c'est à partir des échanges avec le locuteur qu'elle juge si sa participation au groupe de paroles peut être bénéfique ou non, pour lui et/ou pour le groupe.

${ }^{\text {ix }}$ Le fait d'organiser des face-à-face entre victimes et agresseurs nous fait penser à la justice dite réparatrice qui a quatre principaux objectifs : identifier et prendre les actions pour réparer le dommage causé à la victime, impliquer toutes les parties, réinsérer l'auteur et transformer la relation traditionnelle pour rétablir la paix sociale.

${ }^{\mathrm{x}}$ Le CRÉDOC publie son étude annuelle « Baromètre du numérique » réalisée pour le compte du Conseil Général de l'Économie (CGE), de l'ARCEP et de l'Agence du Numérique. Étude 2016 : l'usage du numérique en France par Thomas Coëffé, le 2 décembre 2016.

xi Les hentaï (Manga japonais à caractère pornographique) mettent en scène des « Lolicons » et des « shotacons ».

xii En novembre 2013, François Perea, maître de conférences en sciences du langage à l'université Paul Valéry Montpellier 3, a présenté une Habilitation à Diriger des Recherches sur le thème des "sujets sensibles".

xiii En septembre 2015, Béatrice Damian maître de conférences en sciences de l'information et de la communication à l'Institut d'Eudes Politiques de Rennes a présenté son Habilitation à Diriger des Recherches sur le thème des fantasmes sexuels pour les hommes, par la médiation du corps des femmes.

${ }^{\text {xiv }}$ S. Branca-Rosoff, S. Fleury, F. Lefeuvre, M. Pires, Discours sur la ville. Corpus de Français Parlé Parisien des années 2000 (CFPP2000), http://ed268.univ-paris3.fr/CFPP2000/

${ }^{\mathrm{xv}}$ L'équipe DELIC (Description Linguistique Informatisée sur Corpus, dirigée par Jean Véronis) est une ancienne équipe d'accueil (EA 3779) de l'Université de Provence. Elle s'appelle maintenant TALEP qui est l'acronyme de Traitement Automatique du Langage Écrit et Parlé et désigne l'équipe créée au sein du LIF le 1er janvier 2008 et qui résulte de la fusion de l'ancienne équipe CALN (Compréhension automatique du langage naturel, dirigée par Paul Sabatier) du LIF, et d'une partie de l'équipe DELIC.

${ }^{\text {xvi }}$ Au cours des 3 ans de constitution de corpus, aucune personne de sexe féminin n'a participé aux groupe de parole en se présentant en tant que détentrice et consommatrice de vidéo pédopornographique.

xvii Chaque locuteur est anonymé en conservant toutefois le «genre » du prénom. 
xviii Le Trameur classe par ordre décroissant de fréquence les connecteurs suivants : que (780), mais (236), et (190), quand, (161), si (115)...

xix En 1958, Perelman et Olbrechts-Tyteca ont proposé la définition suivante de l'argumentation : «L'objet de la théorie de l'argumentation est l'étude des techniques discursives permettant de provoquer ou d'accroître l'adhésion des esprits aux thèses qu'on présente à leur assentiment » $(1958: 5)$.

${ }^{\mathrm{xx}} \mathrm{Cf}$. graphe qui représente la répartition des occurrences de mais situé à la page suivante. 